\title{
Descripción y referencia: el lenguaje como posibilidad de veracidad. Un asunto de límites de sistemas-mundo y actos de habla*
}

\author{
FRANCISCO LUIS GIRALDO GUTIÉRREZ** \\ Instituto Tecnológico Metropolitano de Medellín, Colombia \\ franciscogiraldo@itm.edu.co
}

DAVID ALBERTO LONDOÑO VÁSQUEZ *** Institución Universitaria de Envigado, Colombia dalondono@correo.iue.edu.co

Forma de citar este artículo: Giraldo Gutiérrez, F.L., \& Londoño Vásquez, D.A. (2017). Descripción y referencia: el lenguaje como posibilidad de veracidad. Un asunto de límites de sistemas-mundo y actos de habla. Cuadernos de Lingüística Hispánica, (29), 163178. doi: http://dx.doi.org/10.19053/0121053X.n29.2017.5858

* Artículo de reflexión que se desprende del proceso de formación en el Doctorado en Filosofía de la Universidad Pontificia Bolivariana y las investigaciones realizadas alrededor del lenguaje y la pragmática tanto en el Instituto Tecnológico Metropolitano (ITM) como en la Institución Universitaria de Envigado (IUE), Colombia.

** Doctor en filosofía (Escuela de Teología y Filosofía), Universidad Pontificia Bolivariana. Coordinador Maestría CTS +i. Docente Investigador (Facultad de Artes y Humanidades) del Instituto Tecnológico Metropolitano. Código ORCID: orcid.org/0000-00021000-0122

*** Doctor en Ciencias Sociales, Niñez y Juventud (CINDE), Universidad de Manizales. Docente de tiempo completo de la Institución Universitaria de Envigado. Código ORCID: orcid.org/0000-0003-1110-7930 


\title{
Resumen
}

En este artículo desarrollamos el concepto de lenguaje y de cómo él, como acción del hombre en el hombre, al hacerse presente a modo de representación y expresión, tiene la pretensión de validar aquello que conoce -el objeto- y la manera como lo conoce. De esta forma, partimos de concebir el lenguaje como un sistema, una semiótica social y una intención. Mediante el lenguaje y el acto comunicativo de la interlocución, el sujeto determina la veracidad/validez del hecho, la acción: actúa en el sistema y acorde con él, y establece el nivel de correspondencia de la idea -pensada o manifestada- y el objeto, como producto final de su capacidad de hacer y comunicar. Como tesis, planteamos que, independientemente del modo o modos en que se conjuguen los códigos lingüísticos, lo que importa es su posibilidad de veracidad. De igual modo, esos niveles y posibilidades están limitados o ampliados por los sistemas-mundo de los sujetos intervinientes en el acto de habla.

Palabras clave: lenguaje, veracidad, conocimiento, descripción, referencia, sistema-mundo.

\section{Description and Reference: Language as a Possibility for Veracity. A Matter of World-System Limits and Speech Acts}

\begin{abstract}
This article deals with the concept of language, and the way in which, as a manmade artifact, it can be understood as a means of representation and expression, with the pretension of validating the things it does not know -the object- as well as the way to know them. In that way, we draw from the idea of language as a system, a social semiotics and an intention. Through language and the communicative act of interlocution, the subject determines the veracity and validity of a fact. Linguistic action performs in the system and for the system, and establishes the level of agreement between the idea -whether it be thought or made manifest- and the object, as a final product of its ability to act and communicate. Our thesis is that, regardless of the way or ways in which linguistic codes are combined, what really matters is their capacity for veracity. In the same way, these levels and possibilities are limited or amplified by the world-systems in which the speaking subjects are involved.
\end{abstract}

Key words: Language, Veracity, Knowledge, Description, Reference, World-System. 


\section{Description et référence: le langage en tant que possibilité de véracité. Un sujet de limites de systèmes-monde et des actes de parole}

\section{Résumé}

Dans cet article on développe le concept de langage; et comment celui-ci, en tant qu'action de l'homme chez l'homme, au moment d'être présent sous forme de représentation, a la prétention de valider ce qu'il connaît -l'objet- et la manière comme il le connaît. De cette manière, nous partons de la conception du langage en tant que système, une sémiotique sociale et une intention. Au moyen du langage et de l'acte communicatif de l'interlocution, le sujet détermine la véracité/validité du fait, l'action: il agit dans le système et en ayant un accord, il établit le niveau de correspondance de l'idée -pensée et manifestée- et l'objet, en tant que produit final de sa capacité de faire et de communiquer. Comme thèse, nous abordons l'idée que, indépendamment de la manière 0 des manières de conjugaison des codes linguistiques, ce qui compte est leur possibilité de véracité. De même, ces niveaux et possibilités sont limités ou élargis par les systèmes-monde des sujets intervenants dans l'acte de parole.

Mots clés: langage, véracité, connaissance, description, référence, système-monde.

\section{Descrição e referência: a linguagem como possibilidade de veracidade. Um assunto de limites de sistemas-mundo e atos de fala}

\section{Resumo}

Neste artigo desenvolve-se 0 conceito de linguagem e como ela, como ação do homem no homem, ao fazer-se presente a modo de representação e expressão, tem a pretensão de validar aquilo que conhece -0 objeto- e a maneira como o conhece. Desta forma, partimos de conceber a linguagem como um sistema, uma semiótica social e uma intenção. Mediante a linguagem e 0 ato comunicativo da interlocução, o sujeito determina a veracidade/validez do feito, a ação: atua no sistema de acordo com ele, e estabelece o nível de correspondência da ideia -pensada ou manifestada- e o objeto, como produto final de sua capacidade de fazer e de comunicar. Como tese, propomos que, independentemente do modo ou modos em que se conjuguem os códigos linguísticos, o que importa é sua possibilidade de veracidade. De igual modo, esses níveis e possibilidades estão limitados ou ampliados pelos sistemas-mundo dos sujeitos intervenientes no ato de fala.

Palavras chave: linguagem, veracidade, conhecimento, descrição, referência, sistema-mundo. 


\section{Introducción}

Si bien hay una diferencia conceptual entre lengua (idioma), lenguaje (capacidad) y habla (uso individual de la lengua), sería posible señalar que el lenguaje es aquello que el humano es capaz de representar, utilizando las opciones posibles dentro de la lengua y haciéndolo parte de su habla; por tanto, el lenguaje en el hombre sería expresión, manifestación; en suma: estaríamos hablando de comunicación. Lo que varía y ha variado a lo largo de la evolución son las condiciones, los modos y las maneras de representar lo que nos rodea. En otras palabras, "El lenguaje surge en la vida del individuo mediante un intercambio continuo de significados con otros significantes [...] La construcción de la realidad es inseparable de la construcción del sistema semántico en que se halla codificada la realidad" (Halliday, 1982, pp. 9-10). Así, los códigos lingüísticos -regulados por convenciones morfológicas y sintácticas (desde la estructura), y fonéticas-fonológicas y léxico-semánticas (de carácter social) - son los que posibilitan su articulación y tienen aplicabilidad e injerencia en todos los ámbitos de la vida humana.

Ahora bien, conjugándolos, se viabiliza un entendimiento, una acción, un conocimiento entre los intervinientes en el acto lingüístico, que, como causa última, es un acto comunicativo. Es decir, podríamos pensar en el camino que ha recorrido el concepto de lenguaje desde la perspectiva semiológica saussureana pasando por la filosofía analítica de Frege (2005) y Russel (2005), hasta llegar a la filosofía pragmática de Searle, lo cual se podría evidenciar en el acto comunicativo de la interlocución, donde el sujeto debe determinar la veracidad/validez del enunciado. Puesto que, hay una acción propuesta según la lógica del sistema y acorde con él establecen el nivel de correspondencia de la idea - pensada o manifestada - y el objeto, como producto final de su capacidad de hacer (performativa) y comunicar (ilocutiva).

Como tesis, se plantea que independientemente del modo o modos en que se conjuguen los códigos lingüísticos, lo que importa es su posibilidad de veracidad. Por tanto, en este artículo entenderemos una acción comunicativa como veraz/válida cuando el otro o los otros la hayan entendido, así no la acepten o la obedezcan. De igual modo, esos niveles y posibilidades de veracidad están limitados o ampliados por los sistemas-mundo de los sujetos intervinientes en el acto de habla desde una perspectiva searleana, en "como instancia crucial en la generación de sentido [...] en el desocultamiento de la verdad" (Rendón, Londoño y Marín, 2013, p. 182), o para nuestro interés, una construcción del 
contexto de la enunciación y las posibilidades de veracidad. Por tanto, el hecho de que un código lingüístico esté sujeto a convenciones no implica que tenga un nivel de veracidad/ validez: simplemente tiene sentido y significado. Para Beaugrande y Dressler (1997), este último "entendido como la capacidad que tienen las expresiones lingüísticas para ser significantes, y el sentido, entendido como el conocimiento que realmente transmiten las expresiones que aparecen" (p. 409). Ahora está, dicho nivel debe responder a otras circunstancias como lo pragmático.

Para presentar esta tesis de manera razonable, se dará cuenta de tres aspectos: el primero, a modo de antecedentes, de dos líneas de desarrollo de la Filosofía Analítica: los planteamientos de Frege (2005) y Russell (2005); el segundo, el tópico del sistema-mundo; y el tercero, el asunto de los actos de habla, direccionados de manera intencional a cada uno de los aspectos anteriores, en el contexto de los niveles o posibilidades de veracidad/ validez.

\section{Antecedentes del lenguaje como posibilidad de veracidad}

En este aspecto es ineludible, según la historia del desarrollo de la filosofía analítica, tener como punto de partida a dos autores ya clásicos como son Frege y Russell.

\subsection{Elementos para una Teoría de la Referencia de Frege}

Consideramos que quedarnos en la mera estructura sin ahondar en los conceptos, sin indagar y poner en consideración lo planteado, es trabajo perdido. La intención de la filosofía hoy, y del filósofo que la profesa, no es predicar dogmas o establecer sistemas absolutos: solo anhela desarrollar la capacidad de auscultar y darle sentido a la relación entre las cosas por medio del lenguaje. La propuesta de Frege es una reacción desde la filosofía a la mirada saussereana del código lingüístico; no obstante, es consecuente con los planteamientos propuestos por Saussure (1961), quien basa su teoría en la relación significante/significado como elementos indivisibles con respecto al signo.

Veamos, entonces, cómo Frege capitula sus ideas y da respuesta a la pregunta icuál es el sentido de los nombres? (categoría que converge en las propuestas por Niño, 2013); para esto, Frege se centra en establecer la relación entre los nombres, tomando tanto los objetos en sí como los signos de los objetos, esto es, los signos de los mismos nombres, sin tener presente como punto de partida el nombre tal cual se escribe, sino tratando de identificar su significado, examinar qué evoca, a qué lleva, a qué hace referencia el nombre mismo, no las acciones del sujeto que lo posee. 
Con el ánimo de ahondar en la mirada fregeana, el primer elemento para tener en cuenta es la explicación de cómo, a partir de los nombres y el sentido que les demos, se establecen sus posibilidades de relación y las relaciones que posibilitan. De una manera lógica, Frege (2005) establece así veracidades como posibilidades de representación, y aborda este momento con la intención de que "no se confunda la representación que una palabra despierta en un oyente con su sentido o referencia" (p. 33). Reconoce la posibilidad que hay una entidad que se compone de forma indivisible de una representación mental y una imagen 0 un sonido (como anteriormente se mencionó), pero además de que esto se construye en un contexto dado y definido por el interlocutor (usuario).

Cabe preguntar entonces: ¿qué es sentido?, ¿qué es referencia? y ¿qué es representación? En el lenguaje de Frege y en el contexto de la filosofía analítica, se considera que, si se logra dar respuesta a estos tres interrogantes, ya se cumple con el propósito de penetrar en un primer nivel. Según Santamaría (2009), para Frege: "el sentido de una proposición es el pensamiento que es su contenido, y este, a su vez, está determinado por los sentidos de las partes constitutivas de la proposición" (p. 30). El sentido, entonces, es lo que justifica la presencia de las otras palabras en la oración. El sentido no obedece a la mera estructura gramatical, sino a lo que le posibilita el estar ahí, esto es, valida su presencia en esa oración y no en otra. Siguiendo esta línea, Santamaría (2009) precisa:

El propósito de la distinción entre sentido y referencia era el de dar una explicación de los enunciados de identidad no triviales [...]. Hay enunciados tautológicos llamados triviales, como «Napoleón es Napoleón» 0 «El emperador es el Emperador», enunciados obvios, que tienen una veracidad que es necesaria. $Y$ hay también enunciados de identidad no triviales, esto es, enunciados del tipo « $a=b$ » que, como advertimos, suelen ser sintéticos, empíricos y a posteriori. Este tipo de enunciados, a diferencia de los triviales, no son tautológicos, obvios, ni repetitivos, sino, por el contrario, «aumentan el saber» (como lo son los juicios sintéticos) brindándonos más información (p. 30).

En relación con el sentido, se presenta la referencia en los enunciados triviales, como correspondencia entre el nombre y el objeto -individuo-. Los enunciados no triviales "tienen dos características importantes: la primera, que salvaguardan la identidad (referente-individuo); y la segunda, que salvaguardan la informatividad (el sentidoinformación)" (Santamaría, 2009, p. 30). Denota esto, entonces, un primer nivel de veracidad: la correspondencia del nombre -como el referente- y el individuo -como lo referido, de quien se dice algo, aquel que es designado-. En este mismo sentido, se toma la informatividad referida por Santamaría, esto es, la posibilidad de veracidad de la información, que es lo que le da sentido al nombre y, por ende, al individuo. La información, en términos de veracidad, es lo que amplía el conocimiento de lo nombrado: el individuo, 
el sujeto, el objeto o la cosa, dentro de una cultura dada. Esta relación se aprecia cuando Eco (1977) sustenta que:

Cualquier intento de determinar lo que es el referente de un signo nos obliga a definir este referente en términos de una entidad abstracta que no es otra cosa que una convención cultural (entiéndase cultural como especializada). Pero incluso admitiendo que se quiera determinar si es posible, en algunos términos, indicar extensivamente un referente real perceptible con los sentidos, el que identifica el significado con el referente (o quiere hacer depender el valor del signo de la presencia del referente) se ve obligado a separar, de un razonamiento sobre el significado, todos los signos que no pueden corresponder a un objeto real. (p. 119).

Siguiendo con el tema de la veracidad, Frege (2005) lo aborda desde el tipo o tipos de oración que se establezcan y los modos y las posibilidades de relación de los componentes en la misma oración o entre distintas oraciones. Es así como

Hemos visto que a una oración hay que buscarle siempre una referencia, cuando se está interesado en la referencia de sus partes componentes; y esto sucede cuando y solo cuando se da el caso de que nos preguntamos por el valor de la verdad. (p. 35).

Vemos así, cómo lo que importa no es la verdad de la oración, sino el sentido y el significado de lo que la misma oración representa; puesto que hay oraciones que en su formulación son falsas, pero que tienen un amplio sentido para quien las pronuncia y para quien las escucha. Esto es asunto de cómo interactúan las oraciones y del nivel de conocimiento que tengan quien las enuncia y quien las recibe. Cabe mencionar que si bien Frege no lo menciona, en esta situación se requiere de un contexto determinado para poder afianzar la posible veracidad de las oraciones (Marín, 2004), en los términos anteriormente expuestos.

Para ello es necesario tomar algunas ideas desde la pragmática de John Searle (2005b), las cuales nos permiten ver cómo "los nombres propios no son equivalentes a descripciones definidas porque, por ejemplo, llamar a un objeto por su nombre no es un modo de describirlo. Nombrar es una preparación para describir, no un modo de describir" (p. 107). El nombre, aun en su particularidad, se queda corto en lo que respecta a la intención de darle sentido a lo nombrado; de igual manera, «las descripciones no son equivalentes definicionales del nombre, porque son solo contingentemente verdaderas del portador. Pero el nombre no es "verdadero de" el portador: es su nombre» (p. 107). Las propiedades del nombre están referenciadas en lo descrito en el mismo. En términos de las posibilidades de veracidad, con Searle vemos cómo no basta entonces con la referencia: esto es una crítica a la insuficiencia de la propuesta fregeana, que le da un alto valor a la referencia, y por ende a la propuesta saussereana. Según Frege, en la referencia está la 
veracidad sobre el nombre, y es así como para él, el sentido y el significado es lo que le da un nivel de veracidad a la oración, no su misma estructura.

Lo anterior nos lleva a preguntarnos, entonces, si la posibilidad descriptiva a que dé lugar el objeto aumentará el nivel de veracidad del mismo y sobre él, esto es, si a mayor descripción -referencia- del objeto, este tendrá mayor veracidad y, por ende, mayor nivel de conocimiento.

\subsection{Bertrand Russell: sobre la necesidad de describir y la insuficiencia de la referencia}

Russell (2005) presenta, a nuestro modo de comprender, un avance -más que una contradicción- a lo propuesto por Frege. Como se presentó en el numeral anterior, Frege plantea que el sentido de una oración, una proposición o un nombre, se hace evidente por el mero hecho de enunciarlo, referenciarlo. Para Russell, no basta con la referencia: es necesaria la descripción; en este caso, solo la descripción posibilitará la existencia real del objeto, sujeto o hecho en cuestión. De esta manera, anota: "Una descripción puede ser de dos clases: definida 0 indefinida (o ambigua). Una descripción indefinida es una expresión de la forma un tal-y-tal, y una descripción definida es una expresión de la forma el tal-ytal" (p. 50).

La descripción indefinida es ambigua, genérica. En igual estado se encuentra la oración cuando a la pregunta ¿Con quién te encontraste? Se responde: con un hombre, puede ser un hombre cualquiera, no particulariza y, por ende, no posibilita un nivel de realidad/veracidad sobre lo referenciado del sujeto-hombre. Si bien en términos de Frege, la respuesta tiene sentido y se refiere a alguien, en realidad no responde de manera real, con un referente real; por este tipo de deficiencias de la construcción sintáctica en la cual se codifica el sistema desde la gramática (oraciones), la lógica (proposiciones) o la pragmática (enunciados).

Todo esto es un punto de partida con miras a dar cuenta de cómo en ese tránsito del sentido y la referencia propuesto por Frege, y esa necesidad de la descripción planteada por Russell, se trata de establecer las condiciones y modos en que las oraciones, simples 0 complejas, definidas o indefinidas, adquieren un nivel de veracidad, y que, en ese sentido, se hace posible hablar de niveles de lenguaje, de actos de habla, en un sistema-mundo.

Al respecto, los actos de habla son la respuesta a la perspectiva estructuralista de la filosofía del lenguaje. Donde lo importante ya no es lo sistemático de la función, sino que es en verdad lo que se dice en el enunciado, saliéndose de la literalidad y centrándose en 
las potencialidades de combinación comunicativa, las cuales están reguladas por lo social, lo cultural y lo individual.

\section{Sistema-Mundo: fronteras y relaciones del lenguaje}

Como hemos planteado desde el inicio, para nosotros, el lenguaje es comunicación, acción y veracidad. Es decir, "el lenguaje como medio de reflexión sobre las cosas, y el lenguaje como medio de acción sobre las cosas" (Halliday, 1982, p. 10). Para que el lenguaje pueda cumplir con las acepciones anteriores -y las demás que se le pueden otorgar-, es necesario que funcione sistémicamente, esto es, que cumpla con un movimiento metódico dual: de lo inductivo a lo deductivo y viceversa; y que se dé, aunque sea de manera desordenada y caótica, una relación e interdependencia de las partes con el todo. En este estado de cosas metodológicas, el sistema se soporta en unos subsistemas, los cuales, a su vez, tienen autonomía, capacidad de autoorganización y evaluación. Es el caso del lenguaje, como un macrosistema que se soporta en subsistemas, como lo fonético-fonológico, lo morfológico y sintáctico, lo léxico-semántica y lo pragmático. Es decir, el lenguaje como la capacidad de acción (Londoño, Rendón y Marín, 2011) dentro de un sistema consensuado por la comunidad.

Ahora bien, comencemos por decir qué entendemos por sistema-mundo la acción del lenguaje, todo aquello que tiene sentido, que puede ser referenciado, descrito, nombrado. En palabras de Searle (2005a), hablar del sistema-mundo es "responder a la cuestión general de cómo las palabras se relacionan con el mundo" (p. 105), con las cosas, las personas y los objetos que nos rodean. Por tanto, el límite del sistema-mundo es el lenguaje.

Es así como Searle desarrolla su propuesta de sistema-mundo a partir del nombre: de la acción de nombrar las cosas, objetos, sujetos, y su correspondencia con el mismo. Retoma cuestionamientos de la filosofía clásica griega como el diálogo Teeteto de Platón; de igual forma, Searle invita de manera permanente a hacer una valoración de los nombres propios y plantea la tesis inicial de que los nombres propios representan objetos. Veamos su planteamiento:

De acuerdo con la teoría largamente sostenida, los nombres propios simplemente representan objetos sin tener ningún otro sentido o significado que el de representar objetos. Una formulación temprana de esta teoría está en el Teeteto de Platón, y las más sofisticadas versiones modernas de esta idea están en el Tractatus Logico Philosophicus de Wittgenstein y en Philosophy of Logical Atomism de Russell. (p. 105). 
En la línea de los nombres propios hay una herencia de teorías, de las cuales Searle hace referencia, estableciendo lo siguiente:

En la primera teoría los nombres propios son sui generis, y realmente para Platón (en el Teeteto) y Wittgenstein (en el Tractatus) son el vínculo conector especial entre las palabras y el mundo; en la segunda teoría los nombres propios son solo una especie de descripciones definidas disfrazadas: cada una es equivalente en significado a una descripción definida, a la descripción definida que da una formulación explícita de su sentido. De acuerdo con la primera teoría, nombrar es anterior a describir; de acuerdo con la segunda, describir es anterior a nombrar, porque un nombre solo nombra describiendo el objeto que nombra. (p. 107).

Para Searle, lenguaje y mundo se conectan a partir de los nombres. El nombre es considerado sui generis, en tanto que hay una única correspondencia entre el nombre y lo nombrado. Desde nuestro punto de vista, esta teoría, platónica por demás, limita las posibilidades de los nombres y las cosas en un sistema-mundo. Es una teoría reduccionista del lenguaje. Ampliando la segunda teoría, Searle sostiene que:

Según Wittgenstein, el significado de un nombre propio es simplemente el objeto por el que está. Tal vez la formulación más famosa de esta teoría del no-sentido de los nombres propios es la afirmación de Mill de que los nombres propios tienen denotación pero no connotación. Para Mill, un nombre común como «caballo» tiene tanto una connotación como una denotación; connota aquellas propiedades que serían específicas de una definición de la palabra «caballo» y denota todos los caballos. Pero un nombre propio solo denota a su portador. (p. 105).

Decir el caballo no es lo mismo que decir el caballo Rocinante de don Quijote. Vemos así cómo el sistema-mundo del concepto caballo adquiere sentido y referencia cuando decimos el caballo Rocinante de don Quijote, con lo cual se cumple la propuesta de Mill en la cita de Searle en relación con la concepción de dicho autor sobre los nombres propios, en especial la connotación de Rocinante (en algunas comunidades hispanohablantes), a partir de cómo lo describe Cervantes en su obra Don Quijote. Incluso hoy en día podemos decir, aceptar, dar por hecho, que las características de Rocinante, en términos de lo connotativo, se han trasladado a personas, a las cuales, por analogía y símil se les da esos atributos, si bien aquí se identifica un sistema-mundo del nombre, en términos de referencia, sentido, descripción, veracidad y posibilidad.

Al respecto, la palabra caballo, denotativamente, no le agrega nada al concepto, simplemente lo referencia, siempre y cuando a quien se le comunique -el interlocutoresté en el mismo sistema-mundo, esto es, siempre que los agentes del lenguaje identifiquen 
y apliquen los mismos códigos lingüísticos. Esta aseveración es coherente con la pragmática. Searle da elementos para ampliar lo anterior:

Así, si un nombre propio aparece en un enunciado existencial, parece que debe tener algún contenido conceptual o descriptivo. Pero si tiene un contenido descriptivo, entonces parece que la teoría de Frege debe ser correcta, porque: ¿qué podría ser ese contenido descriptivo salvo el sentido del nombre propio? De este modo, la ocurrencia de los nombres propios en enunciados existenciales añade otra grave dificultad para los teóricos del no-sentido. Sin embargo lo peor aún no ha llegado. (pp. 107-108).

Para Searle,

Lo peor se hace evidente si en un momento de destrucción del objeto, cosa 0 desaparición de la persona, o especie, o cosa que lleva el nombre, se tenga que desaparecer -diría yo de manera forzada- el significado de la palabra, el concepto" (p. 200).

El sistema-mundo, en vez de ampliarse exponencialmente, sufriría el efecto contrario: se vería reducido a su mínima expresión. Vemos cómo las características, condiciones y modos en que el nombre adquiere un nivel de veracidad, de sentido, de referencia, están en el sistema-mundo; sin embargo, en igual medida, lo que connota el nombre, lo referido, lo descrito, adquiere veracidad/validez en los actos de habla, esto es, en la interlocución, en la interacción comunicativa.

\section{Actos de habla: interlocución y posibilidades de veracidad del lenguaje}

Para hablar hay que saber, y para saber hay que leer y observar, lo cual nos posibilita comunicar de manera efectiva. Los sujetos nos comunicamos con otros dentro de un contexto, el cual probablemente se deba compartir para poder construir un acto comunicativo. De igual forma, es necesario compartir la lengua (o el sistema), en pro de codificar y decodificar, de modo mínimo o amplio. Por ello,

Lo que constituye la unidad básica de la comunicación lingüística es la producción de la instancia en la realización del acto de habla. Para establecer más precisamente este punto: la producción de la oración-instancia bajo ciertas condiciones es el acto ilocucionario, y el acto ilocucionario es la unidad mínima de la comunicación lingüística. (Searle, 2005a, p. 432). 
Es decir, en los actos de habla ya no se piensa en términos de oraciones o unidades lingüísticas (lo locucionario), ni en los efectos que esto tenga en el interlocutor (lo perlocutivo), sino en la intención real de lo que se dice y el cómo se dice (lo ilocucionario). En relación con el sistema-mundo, a este le corresponden unos códigos lingüísticos, no de manera exclusiva, pero sí de forma contextualizada y válida al momento del acto comunicativo en que se hacen presentes. Al respecto, Searle (2005a) sostiene que:

En una situación de habla típica que incluye un hablante, un oyente y una emisión del hablante, existen muchos géneros de actos asociados con la emisión del hablante. El hablante habrá movido característicamente su mandíbula y su lengua y habrá producido ruidos. Además, habrá realizado característicamente algunos actos pertenecientes a la clase que incluye informar 0 irritar 0 aburrir a sus oyentes. (p. 431).

Vemos cómo en los actos de habla, los códigos lingüísticos se amplían a través de expresiones como arquear las cejas, fruncir el ceño, bostezar cuando el otro habla, no mirar a quien habla, entre otros gestos diarios, participan en la construcción de sentido y significado, son determinantes al momento de validar una información, de darle un nivel de credibilidad a lo que dice el otro y un nivel de aceptación y entendimiento en quien escucha. Según Searle (2005a):

Los actos de habla se realizan característicamente en la emisión de sonidos 0 en la producción de marcas. ¿Cuál es la diferencia entre solamente emitir sonidos 0 hacer marcas y realizar un acto de habla? Una diferencia consiste en que de los sonidos o marcas que una persona hace en la realización de un acto de habla se dice característicamente que tienen significado, y una segunda diferencia relacionada consiste en que característicamente se dice que una persona quiere decir algo mediante esos sonidos o marcas. (p. 437).

Teniendo lo anterior como referente, es necesario decir que plantear los actos de habla en Searle es hablar de actos ilocucionarios, quien a su vez toma el concepto de los enunciados de acción o enunciados performativos de Austin (1962), en los cuales se toma parte por algo 0 alguien, dependiendo de la acción implícita en la ilocución. Pongamos por caso una orden, un regaño, un consejo, una censura, un saludo. No solo esto; en igual contexto, es claro que para Searle (2005a):

En la realización de un acto ilocucionario el hablante intenta producir un cierto efecto, logrando que el oyente reconozca su intención de producir ese efecto, y además, si está usando las palabras literalmente, intenta que este reconocimiento se logre en virtud del hecho de que las reglas para el uso de las expresiones que emite asocien las expresiones con la producción de ese efecto. (pp. 439-440). 
Es decir, en la realización de un acto ilocucionario (acto que se realiza diciendo algo), el hablante intenta producir un cierto efecto, logrando que el oyente reconozca su intención de producir ese efecto (actos asertivos, directivos, compromisorios, declarativos o expresivos), y además, si está usando las palabras literalmente, intenta que este reconocimiento se logre en virtud del hecho de que las reglas para el uso de las expresiones que emite asocien las expresiones con la producción de ese efecto.

La anterior es una propuesta aceptada en la pragmática, y esta aceptación ha permitido ver no solo en los elementos constitutivos del lenguaje, sino en los interlocutores y el contexto una posibilidad de interacción social. Al respecto, Halliday (1982) afirma que:

El lenguaje simboliza activamente el sistema social, representando metafóricamente en sus patrones de variación la que caracteriza a las culturas humanas; es lo que permite a la gente jugar con la variación en el lenguaje, utilizándola para crear significados de tipo social. (p. 11).

Por ello es que probablemente cada uno de los elementos socializadores y socializables recurren al lenguaje para poder hacerlo. Aquí los discursos aparecen como una opción no solo de interacción social, sino también de construcción de realidades (o al menos de afectación sobre ellas). En otras palabras, elementos ideológicos, identitarios y sociales están concatenados discursivamente (Van Dijk, 1999). Por tanto, nos atrevemos a proponer que el efecto del lenguaje en los sistemas-mundo es incalculable, pero los niveles de veracidad sí son identificables a través de la fuerza ilocucionaria:

Desde un punto de vista semántico podemos distinguir en la oración entre el indicador proposicional y el indicador de fuerza ilocucionaria. Esto es, para una extensa clase de oraciones usadas para realizar actos ilocucionarios, podemos decir para los propósitos de nuestro análisis que la oración tiene dos partes (no necesariamente separadas), el elemento indicador de la proposición y el dispositivo indicador de la función. El dispositivo indicador de la función muestra cómo debe ser tomada la proposición o, dicho de otra manera, qué fuerza ilocucionaria ha de tener, esto es, qué acto ilocucionario está realizando el hablante al emitir la oración. (Searle, 2005, p. 436).

En este orden de ideas, el éxito del proceso comunicativo no radica solo en el proceso de decodificiación lingüística de un enunciado, es necesario que el hablante tenga la capacidad de inferir cuál significado quiso dar su interlocutor. De igual forma que reconozca el contexto de comunicación en el que se encuentra; "puesto que, diversos enunciados con el mismo contenido proposicional pueden diferir en su fuerza ilocucionaria, según el contexto de comunicación y por supuesto según la intención de enunciación o finalidad del hablante" (Puerta y Sánchez, 2012, p. 102). 
Por tanto, la descripción y la referencia como posibilidad de veracidad en el lenguaje, permitiría dar valor a las Máximas Conversacionales de Grice (1975): cantidad (no digas más ni menos de lo que se necesita), calidad (no digas algo que no sepas que es cierto), modo (dilo de la forma más adecuada) y pertinencia (que lo que digas aporte a la conversación). No obstante, el interlocutor difícilmente podría cumplir las máximas en algunos enunciados, dado a las interacciones que se tejen en el ámbito social e interactivo de las comunidades. En otras palabras, "el lenguaje tiene que expresar nuestra participación, como hablantes, en la situación del discurso" (Halliday, 1982, p. 34); los papeles que asumimos nosotros mismos y que imponemos a otros; nuestros deseos, sentimientos, actitudes y juicios hacen parte de ese proceso descriptivo y referencial, en el marco de un acto comunicativo.

\section{A modo de conclusión}

La complejidad se acrecienta si, desde lo presentado por Santamaría (2009), ese aire de familia no es único, no mantiene una única línea de desarrollo, tiene divisiones que ponen en evidencia un referente de partida y un lugar de llegada. El primero es el atomismo lógico; el segundo, la filosofía del lenguaje ordinario. Un camino que en apariencia es corto, pero cuyo campo de exploración no lo es.

Teniendo en cuenta lo anterior, podríamos señalar que Frege da elementos para entender la descripción como uno de los constructos de la significación y el sentido que permiten aumentar el nivel de veracidad del objeto; puesto que, parece ser que a mayor descripción del objeto, este tendrá mayor veracidad y, por ende, mayor nivel de conocimiento, pero adopta un marco de interpretación posible a través de la contribución de Russell, en relación con la referencia en el proceso descriptivo. Dicho proceso, probablemente, en la actualidad se podría trabajar desde la pragmática, más que desde la gramática o la lógica.

Por ello, es viable resaltar que, se ha hecho la tarea con un punto de partida: Frege y Russel, mediados por Searle, con miras a establecer los niveles de veracidad de ambas propuestas, y de cómo dichas propuestas adquieren un mayor grado de veracidad según el sistema-mundo donde sean vistas. De igual manera, son los sujetos que intervienen en los actos ilocucionarios los que en el límite 0 amplitud del sistema-mundo, en últimas validan los códigos lingüísticos y actúan según el nivel de entendimiento de dichos códigos.

Finalmente, hay elementos sociales, culturales, cognitivos, psicológicos y lingüísticos que juegan roles intercambiarios entre los participantes de un acto comunicativo, determinando acciones descriptivas y referenciales que están sujetas a posibles connotaciones y, en algunos casos, esto afecta el proceso interpretativo de las mismas. No obstante, la relación de veracidad/validez puede ser adecuada si dentro de las posibilidades, estas son aceptadas por los participantes. 


\section{Referencias bibliográficas}

Austin, J. (1962). How to Do Things With Words (2nd edition). Cambridge: Harvard University Press.

Beaugrande, R., \& Dressler, W. (1997). Introducción a la Lingüística del texto. Barcelona: Ariel Lingüística.

Eco, U. (1977). Tratado de Semiótica General. Barcelona: Lumen.

Frege, G. (2005). Sobre sentido y referencia. En: L. Valdés. La Búsqueda de significado. Lecturas de filosofía del lenguaje (pp. 29-49). Madrid: Tecnos.

Grice, P. (1975). Logic and Conversation. En: P. Grice (1989). Studies in the Way of Words. Boston: Harvard University.

Habermas, J. (1989). Teoría de la acción comunicativa. Complementos y estudios previos. Madrid: Cátedra.

Halliday, M.A.K. (1982). El lenguaje como semiótica social. México: Fondo de Cultura Económica.

Londoño, D., Rendón, J., \& Marín, G. (2011). Las utopias racionalista y deliberativa: El sujeto moral kantiano y su tripartición habermasiana como formas optimistas de la política. Revista Virtual Universidad Católica del Norte, 33. Medellín: Universidad Católica del Norte.

Marín, M. (2004). Lingüística y enseñanza de la lengua. Buenos Aires: AIQUE.

Niño, V. (2013). Semiótica y lingüística: fundamentos. Bogotá: ECOE Ediciones.

Platón. (1988). En: Parménides, Teeteto, Sofista, Político. Madrid: Editorial Gredos. S. A.

Puerta, C., \& Sánchez, L. (2012). Análisis lingüístico de la retroalimentación en educación virtual. En: A. Sánchez (Ed.). El Análisis lingüístico como estrategia de alfabetización académica. Santa Rosa de Osos: Fundación Universitaria Católica del Norte.

Rendón, J., Londoño, D., \& Marín, G. (2013). El papel postmoderno de la identidad personal: El lugar de la subjetividad en el giro hermenéutico. Revista Virtual Universidad Católica del Norte, 40. Medellín: Universidad Católica del Norte.

Russell, B. (2005). Descripciones. En: L. Valdés. La búsqueda del significado. Lecturas de Filosofía del lenguaje (pp. 50-60). Madrid: Tecnos.

Santamaría, F. (2009). Hacer mundos: El nombrar y la significatividad. una investigación desde la Filosofía Analítica. Bogotá: Universidad Santo Tomás.

Saussure, F. (1961). Curso de lingüística general. Buenos Aires: Losada. 
Searle, J. (2005a). ¿Qué es un acto de habla? En: L. Valdés. La Búsqueda de significado. Lecturas de filosofía del lenguaje (pp. 431-447). Madrid: Tecnos.

Searle, J. (2005b). Nombres propios y descripciones. En: L. Valdés. La Búsqueda de significado. Lecturas de filosofía del lenguaje (pp. 105-115). Madrid: Tecnos.

Van Dijk, T. (1999). Ideología. Barcelona: Gedisa.

Wittgenstein, L. (2009). Tractatus logico-philosophicus. Madrid: Editorial Gredos S, S.A. 